\title{
Hedgehog signaling in the neural crest cells regulates the patterning and growth of facial primordia
}

\author{
Juhee Jeong, ${ }^{1}$ Junhao Mao, ${ }^{1}$ Toyoaki Tenzen, ${ }^{1}$ Andreas H. Kottmann, ${ }^{2,3}$ and Andrew P. McMahon ${ }^{1,4}$ \\ ${ }^{1}$ Department of Molecular and Cellular Biology, Harvard University, Cambridge, Massachusetts 02138, USA; ${ }^{2}$ Howard \\ Hughes Medical Institute, Department of Biochemistry and Molecular Biophysics, Center for Neurobiology and Behavior, \\ Columbia University, New York, New York 10032, USA
}

Facial abnormalities in human $S H H$ mutants have implicated the Hedgehog (Hh) pathway in craniofacial development, but early defects in mouse $S h h$ mutants have precluded the experimental analysis of this phenotype. Here, we removed Hh-responsiveness specifically in neural crest cells (NCCs), the multipotent cell type that gives rise to much of the skeleton and connective tissue of the head. In these mutants, many of the NCC-derived skeletal and nonskeletal components are missing, but the NCC-derived neuronal cell types are unaffected. Although the initial formation of branchial arches (BAs) is normal, expression of several Fox genes, specific targets of Hh signaling in cranial NCCs, is lost in the mutant. The spatially restricted expression of Fox genes suggests that they may play an important role in BA patterning. Removing Hh signaling in NCCs also leads to increased apoptosis and decreased cell proliferation in the BAs, which results in facial truncation that is evident by embryonic day 11.5 (E11.5). Together, our results demonstrate that Hh signaling in NCCs is essential for normal patterning and growth of the face. Further, our analysis of Shh-Fox gene regulatory interactions leads us to propose that Fox genes mediate the action of Shh in facial development.

[Keywords: Craniofacial development; Shh; Fox genes; branchial arch; neural crest; mouse]

Supplemental material is available at http://www.genesdev.org.

Received January 28, 2004; revised version accepted March 12, 2004.

The neural crest cells (NCCs) are a population of highly migratory multipotent precursors that arise at the junction between the prospective neural tube and epidermis during early stages of vertebrate development (Gammill and Bronner-Fraser 2003). Their diverse fates include several cell types within the peripheral nervous system, melanocytes, endocrine cells, and most of the bone, cartilage, and connective tissue of the face and skull (Helms and Schneider 2003; Santagati and Rijli 2003). NCCs that contribute to the face originate from the caudal forebrain, midbrain, and rostral hindbrain. These cranial neural crest cells (CNCCs) follow well defined paths to the ventrolateral side of the head, where they populate the mesenchyme of the facial primordia (called ectomesenchyme to distinguish it from the mesodermal mesenchyme) such as the frontonasal prominence (FNP) and branchial arches (BAs, also known as pharyngeal arches; Osumi-Yamashita et al. 1994; Köntges and Lumsden

${ }^{3}$ Present address: Columbia Genome Center, Columbia University, New York, NY 10032, USA.

${ }^{4}$ Corresponding author.

E-MAIL amcmahon@mcb.harvard.edu; FAX (617) 496-3763.

Article and publication are at http://www.genesdev.org/cgi/doi/10.1101/ gad.1190304.
1996). Once positioned, the CNCCs proliferate and differentiate into distinct craniofacial elements (Helms and Schneider 2003; Santagati and Rijli 2003).

The ectomesenchyme cells need positional cues in order to generate the elaborate structures of the mature face (Helms and Schneider 2003; Santagati and Rijli 2003). Although the classic prepatterning model states that the fate of a CNCC is determined prior to its $\mathrm{mi}$ gration from the neural tube according to the rostrocaudal level of origin (Noden 1983), a number of reports have demonstrated plasticity within CNCCs and the importance of signals provided by nonneural crest components of the facial primordia, that is, the epithelium and the mesodermal mesenchyme, in craniofacial patterning (Ferguson et al. 2000; Trainor and Krumlauf 2000; Lee et al. 2001; Couly et al. 2002; Tranior et al. 2002; Hu et al. 2003).

Whatever the initiating mechanism is, execution of a specific differentiation program within CNCCs is likely to require modulating the activity of transcriptional regulators. Several families of transcription factors are expressed in the ectomesenchyme, including Hox, Dlx, Msx, Hand, Pax, Prx, and Fox genes (Depew et al. 2002a). For inter-BA patterning, Hoxa2 has been shown to be necessary (in mouse) and sufficient (in chick and frog) to 
confer the second BA as opposed to the first BA fate (Gendron-Maguire et al. 1993; Rijli et al. 1993; Grammatopoulos et al. 2000; Pasqualetti et al. 2000). On the other hand, the intra-BA patterning of the first BA into maxillary (MXA, upper jaw) and mandibular (MNA, lower jaw) arch components appears to depend on the combination of $D l x$ genes expressed in each structure (Beverdam et al. 2002; Depew et al. 2002b).

Sonic hedgehog ( $\mathrm{Shh}$ ) is expressed in the epithelium of facial primordia (Echelard et al. 1993). Given its well established roles in patterning of other organs (McMahon et al. 2003), Shh is a good candidate for one of the molecules that pattern the facial mesenchyme. Indeed, mutations in human $\mathrm{SHH}$ are responsible for a subset of cases of holoprosencephaly, the congenital defect characterized by forebrain and facial abnormalities (Roessler et al. 1996). Unfortunately, however, mouse Shh mutants have early defects in the axial mesendoderm, which is required for normal organization of the head region (Chiang et al. 1996; Pera and Kessel 1997). The resulting severe deficiencies in head structures have precluded the analysis of possible later roles for Shh in the regulation of facial morphogenesis (Chiang et al. 1996). Efforts have been made to circumvent this problem by manipulating chick embryos after the establishment of the basic head plan. Loss-of-function approaches using function-blocking anti-Shh antibody and gain-of-function approaches by ectopic application of Shh protein have established the importance of Shh for survival and proliferation of ectomesenchyme cells (Ahlgren and Bronner-Fraser 1999; Hu and Helms 1999). However, the local and/or transient natures of these manipulations may not have revealed the full extent of Shh action in craniofacial development. Furthermore, adding or blocking the diffusible ligand may affect multiple tissues in the facial primordia, that is, the epithelium, mesodermal mesenchyme, and the ectomesenchyme. Therefore, it remains unclear whether there is a direct requirement for Hh signaling within ectomesenchyme to make craniofacial elements, and if this is the case, whether Hh signaling may contribute to molecular patterning of the facial mesenchyme in addition to promoting cell survival and proliferation. Here, we demonstrate that direct $\mathrm{Hh}$ signaling to the CNCCs is essential for formation of most craniofacial structures. We propose a model in which $\mathrm{Hh}$ signaling patterns the facial mesenchyme via combinatorial expression of several Forkhead transcription factors (encoded by Fox genes).

\section{Results}

Expression of Shh and Ptch1 during normal craniofacial development

To obtain a detailed understanding of Shh signaling during normal craniofacial development, we examined the expression patterns of Shh and its transcriptional target Patched1 (Ptch1; Goodrich et al. 1996) in relation to the distribution of CNCCs in the FNP and BAs. CNCCs were labeled using Wnt1-Cre, an NCC-specific Cre transgene (Supplementary Fig. S1A; Danielian et al. 1998; Chai et al. 2000; Jiang et al. 2000), and the R26RLacZ Cre reporter line (Soriano 1999). At embryonic day 9.5 (E9.5) and E10.5, Shh is expressed in three epithelial populations in the developing face, that is, the ventral forebrain neuroepithelium (arrow in Fig. 1A), the oral ectoderm (arrow in Fig. 1F), and the pharyngeal endoderm (arrowheads in Fig. 1E,F), but is absent from the mesenchyme (Fig. 1M,P,S). Expression of Ptch1 indicates that Shh signaling occurs in both the epithelium and underlying mesenchyme (Fig. $1 \mathrm{C}, \mathrm{D}, \mathrm{G}, \mathrm{H}, \mathrm{N}, \mathrm{Q}, \mathrm{T}$ ). In the MXA and FNP, only the medial half of the mesenchyme is subject to Hh signaling (Fig. 1H,Q). On the other hand, Ptch1 expression extends along the entire mediolateral (proximodistal) axis of the caudal half of the MNA, although its expression is dorsally restricted except at the midline (Fig. 1H,T). Additional sites of Shh production appear at E12.5, including the ventral nasal pit (arrow in Fig. 1J) and tongue epithelium (Fig. 1I,J,V, see arrowhead in J). A significant part of the nasal, oral, and tongue mesenchyme receives $\mathrm{Hh}$ signaling at this stage (Fig. $1 \mathrm{~K}, \mathrm{~L}, \mathrm{~W})$. The mesenchyme expressing Ptch1 contains a high density of CNCCs at all three stages examined (Fig. $1 O, R, U, X)$, suggesting that direct $H$ signaling to this population of cells may have functional importance.

In addition to Shh, another member of $\mathrm{Hh}$ family genes, Indian hedgehog (Ihh), has been implicated in craniofacial development; Ihh mutants have mild craniofacial abnormalities (St-Jacques et al. 1999). However, no Ihh expression is detected in the head by in situ hybridization until E12 (Supplementary Fig. S2; Kronmiller and Nguyen 1996). Thus, Shh is exclusively responsible for the $\mathrm{Hh}$ signaling in the face prior to E12.

Hh signaling in CNCCs is necessary for the formation of most of the head skeleton

In order to understand the role Hh signaling plays in the ectomesenchyme during craniofacial development, we removed Hh-responsiveness from the entire neural crest lineage by crossing mice harboring Wnt1-Cre with those that are conditionally null for Smoothened (Smo), an obligatory and cell-autonomous component of Hh signal transduction in responding tissue (Supplementary Fig. S1B; Zhang et al. 2001; Wnt1-Cre;Smo ${ }^{n / c}$ ). Conversely, we performed Hh signaling gain-of-function experiments within CNCCs by Cre-mediated activation of a dominant active form of Smo (SmoM2; Xie et al. 1998) in Wnt1-Cre;R26SmoM2 embryos (see Materials and Methods; Supplementary Fig. S1C).

Wnt1-Cre; $\mathrm{Smo}^{n / c}$ embryos appear indistinguishable from the wild types until E10.5, when they have slightly smaller FNPs and MNAs (Fig. 2A,B). The growth deficiency of mutant facial primordia is very obvious by E12.5, and as a result, mice are born with a dramatically truncated face (Fig. 2C-F). The fact that no defect is apparent until more than $24 \mathrm{~h}$ after the migration of CNCCs into the FNP and first BA is completed (E9; Depew et al. 2002a) suggests that earlier events such as 

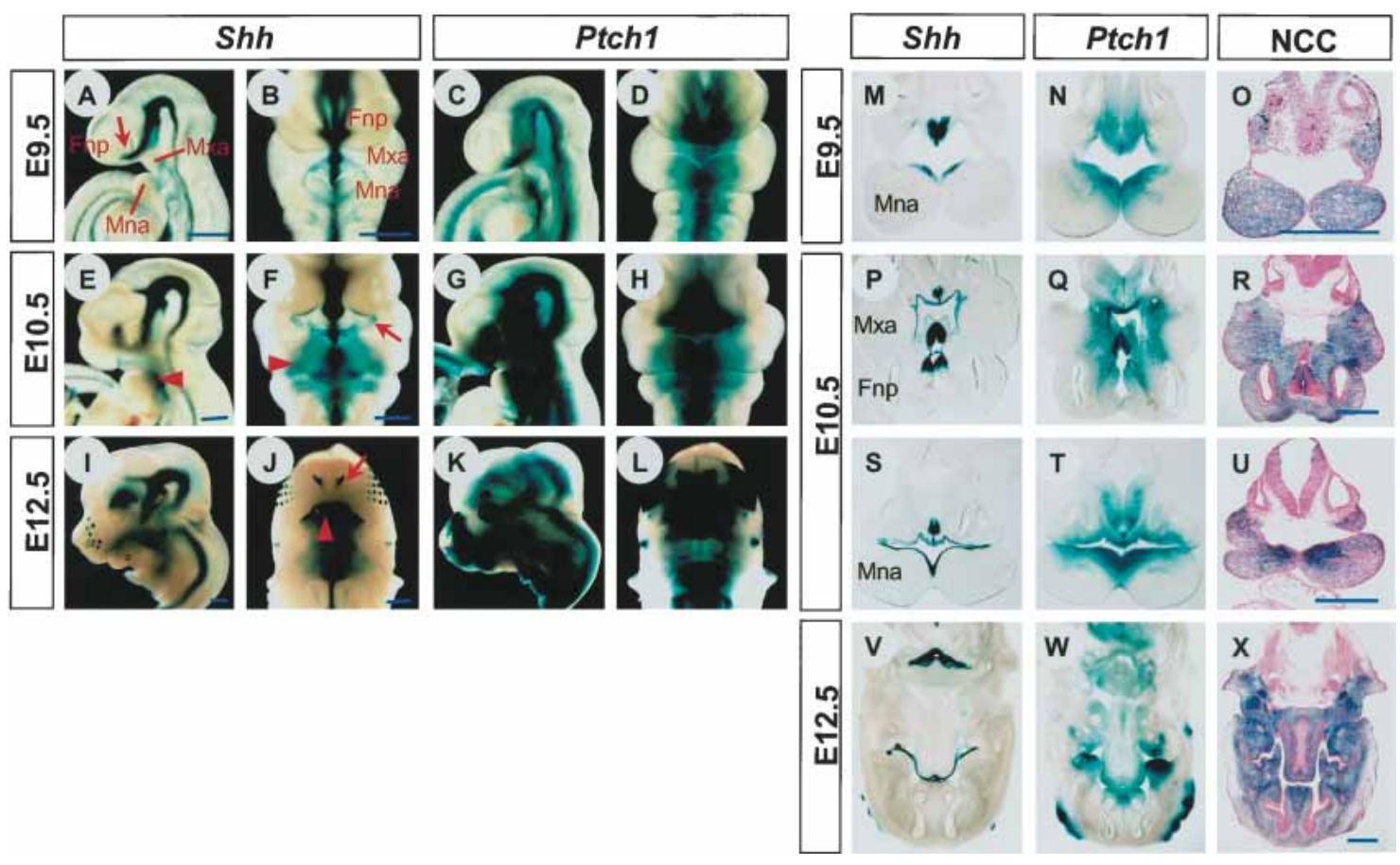

Figure 1. Expression of Shh and Ptch1 in relation to facial development in the mouse embryo. Lateral views $(A, E, I)$ and frontal views $(B, F, J)$ of whole-mount $\beta$-galactosidase staining of heads from $S h h^{1 a c Z /+}$ embryos. Lateral views $(C, G, K)$ and frontal views $(D, H, L)$ of whole-mount $\beta$-galactosidase staining of heads from Ptch1 $1^{1 a c Z /+}$ embryos. $(M-X)$ Transverse sections through the heads of $S h h^{1 a c Z /+}$ $(M, P, S, V)$, Ptch1 $^{1 a c Z /+}(N, Q, T, W)$, and Wnt1-Cre;R26R-LacZ $(O, R, U, X)$ embryos. Stages of development as indicated in each panel. Bar, $0.5 \mathrm{~mm}$.

NCC-generation and migration are not affected. Three lines of evidence further support this argument. First, $A P-2$, a marker for NCCs (Mitchell et al. 1991), is expressed normally in the mutants at E9.25 (data not shown). Second, lineage labeling of NCCs by Wnt1-Cre and R26R-LacZ indicates that NCCs are present in the mutant facial mesenchyme at E10.5, and their gross distribution resembles that observed in wild-type embryos (Fig. 2G,H). Third, cranial and trunk ganglia, which also originate from the neural crest, develop normally, arguing against any early and generalized defects in the neural crest (Fig. 2I,J; data not shown). Together, these data suggest that the craniofacial phenotype of Wnt1-Cre; $S m o^{n / c}$ embryos reflects the requirement for Hh signaling in postmigratory CNCCs within the facial primordia.

Analysis of skeletal preparations of E18.5 Wnt1-Cre; $\mathrm{Smo}^{n / c}$ heads reveals the extensive loss of craniofacial structures (Fig. 2K-U). Rostrally, the nasal capsule is normal, but the nasal bone is hypoplastic and the nasal septum is incomplete (Fig. 2K,L). The lacrimal bone is absent or appears as a tiny fragment, and the mesethmoid is missing (data not shown). Premaxilla and maxilla retain their lateral-most parts only, and the vomer has completely disappeared (Fig. 2M,N). More caudally, the jugal and squamosal bones are reduced, and the palatine and pterygoid are absent (Fig. 2M,N). The skull base is also severely affected; the orbitosphenoid, presphenoid, and rostral half of the basisphenoid are missing, and the alisphenoid is reduced (Fig. 2M,N). In contrast, the three occipital bones (basi-, exo-, supra-), which derive from paraxial mesoderm, are normal (Fig. $2 \mathrm{~K}, \mathrm{~L}$ ). The defect of the skull vault is limited to the decreased size of the neural crest-derived frontal bone. Parietal and interparietal bones, which are of mesoderm origin, are unaffected (Fig. 2K,L). The dentary is reduced in its length, but the lamina is thicker than the wild type (Fig. 2O-Q). The proximal part is affected less, based on the relatively normal coronoid, condylar, and angular processes. In fact, the inner layer of the lamina possesses an extra condylar process (cdp* in Fig. 2Q), resulting in the partial duplication of the dentary. Meckel's cartilage is hypoplastic and short. In the ear region, the otic capsule appears normal, but the elements associated with it, that is, stapes, incus, malleus, styloid process, gonial bone, and the tympanic ring, are all absent (Fig. 2R,S). The three hyoid elements (basi-, cerato-, thyro-) are missing, as is the thyroid cartilage (Fig. 2T,U). Instead, three to six fragments of cartilage are visible in their places. Arytenoid cartilage, cricoid cartilage, and the tracheal rings posterior to them are intact (Fig. 2T,U). The head skeleton phenotype of Wnt1-Cre;Smo ${ }^{n / c}$ embryos is summarized in Figure 2X. The cross-reference of these defects with the origin of each element (Fig. 2W) reveals that all of the neural crest-derived elements, but none of the mesodermal ones, are affected in the mutants.

In contrast to the loss of Hh signaling, activation of the Hh pathway in CNCCs (Wnt1-Cre;R26SmoM2) causes a mild hyperplasia of the facial processes at E10.5 (Supple- 
Jeong et al.

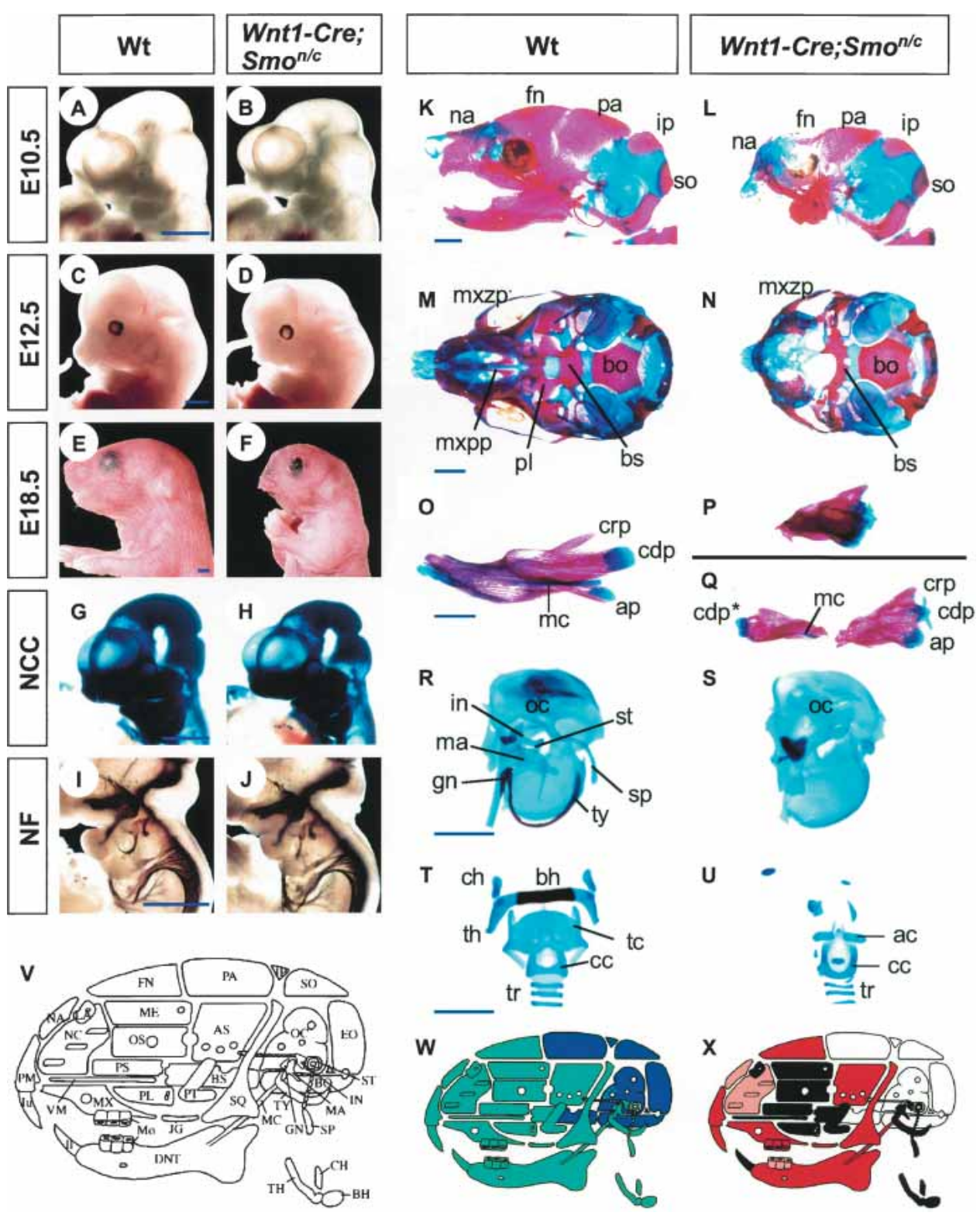

Figure 2. Craniofacial phenotypes of Wnt1-Cre;Smo ${ }^{n / c}$ embryos. $(A-F)$ Heads of wild-type $(A, C, E)$ and Wnt1-Cre;Smo ${ }^{n / c}(B, D, F)$ embryos at the stages indicated. $(G, H)$ Whole-mount $\beta$-galactosidase staining of heads from Wnt1-Cre;R26R-LacZ (G) and Wnt1$\mathrm{Cre} ; \mathrm{Smo}^{n / c} ; R 26 \mathrm{R}-\mathrm{LacZ}(H)$ embryos at E10.5. $(I, J)$ Facial ganglia labeled by whole-mount immunostaining for neurofilament in wildtype $(I)$ and Wnt1-Cre;Smo ${ }^{n / c}(J)$ embryos at E10.5. $(K-U)$ Skeletal preparations of heads from wild-type $(K, M, O, R, T)$ and Wnt1Cre; $S_{m o}{ }^{n / c}(L, N, P, Q, S, U)$ embryos at E18.5. $(K, L)$ Lateral views. $(M, N)$ Skull bases with dentaries removed. (O-Q) Dentaries. In $Q$, the inner and outer layers of the mutant dentary have been separated to show Meckel's cartilage. $(R, S)$ Otic capsules and the associated elements. $(T, U)$ Hyoid and laryngeal cartilages. $(V-X)$ Schematics of head skeleton. $(W)$ Green indicates skeletal elements of NCC origin; blue indicates skeletal elements of mesodermal origin. $(X)$ Pink indicates mildly reduced or malformed in Wnt1-Cre;Smo ${ }^{n / c}$ embryos; red indicates severely reduced in Wnt1-Cre;Smo ${ }^{n / c}$ embryos; black indicates absent in Wnt1-Cre;Smo ${ }^{n / c}$ embryos. (AC) Arytenoid cartilage; (AP) angular process; (AS) alisphenoid; (BH) basihyoid; (BO) basioccipital; (BS) basisphenoid; (CC) cricoid cartilage; (CDP) condylar process; (CH) ceratohyoid; (CRP) coronoid process; (DNT) dentary; (EO) exoccipital; (FN) frontal; (GN) gonial; (Il) lower incisor; (IN) incus; (IP) interparietal; (Iu) upper incisor; (JG) jugal; (LA) lacrimal; (MA) malleus; (MC) Meckel's cartilage; (ME) mesethmoid; (Mo) molar; (MX) maxilla; (MXPP) palatal process of maxilla; (MXZP) zygomatic process of maxilla; (NA) nasal bone; (NC) nasal cartilage; (NF) neurofilament; (OC) otic capsule; (OS) orbitosphenoid; (PA) parietal; (PL) palatine; (PM) premaxilla; (PS) presphenoid; (PT) pterygoid; (SO) supraoccipital; (SP) styloid process; (SQ) squamosal; (ST) stapes; (TC) thyroid cartilage; (TH) thyrohyoid; (TR) tracheal rings; (TY) tympanic ring; (VM) vomer. Bar, $1 \mathrm{~mm}$. $V-X$ were modified with permission from Depew et al. (1999). (C) 1999 The Company of Biologists Limited.

mentary Fig. S3A,B; see also Fig. 5, below, for frontal views). From E12.5, the gross organization of the face is disrupted (Supplementary Fig. S3C-F), and most of the head skeleton fails to form (Supplementary Fig. S3I,J) 
even though CNCCs are clearly present in the facial primordia (Supplementary Fig. S3G,H). The missing skeletal elements include the mesodermal components of the skull vault, which is likely to be a secondary phenotype resulting from ectopic $\mathrm{Hh}$ signaling in the dorsal neural tube and subsequent overgrowth of the brain. To ensure that our analysis explored the direct actions of Shh signaling in CNCCs, we restricted further analysis of these gain-of-function mutants up to E10.5.

Hh signaling regulates the expression of Fox genes in the ectomesenchyme

The complete loss of many facial elements observed in Wnt1-Cre; $\mathrm{Smo}^{n / c}$ embryos is consistent with a role for Shh in specification of certain neural crest-derived structures. We therefore investigated the possibility that $\mathrm{Hh}$ signaling influences the developmental fate of the ectomesenchyme cells by regulating regional gene activity within facial primordia. Transcriptional profiling of Shh mutant and wild-type heads at E10.5 has identified a number of genes whose expression is modulated by the Hh pathway (T. Tenzen and A. McMahon, unpubl.). Notable among this group are several members of the Fox family transcription factors, which share a conserved DNA binding domain (forkhead domain). Fox members play diverse roles in vertebrate embryogenesis and are central to the control of adult metabolism (Carlsson and Mahlapuu 2002). We examined the expression of five Fox genes, Foxc2 (Miura et al. 1993), Foxd1 (Hatini et al. 1994), Foxd2 (Sasaki and Hogan 1993), Foxf1 (Clevidence et al. 1994), and Foxf2 (Miura et al. 1998), in wild-type, Wnt1-Cre;Smo ${ }^{n / c}$, and Wnt1-Cre;R26SmoM2 facial primordia. Loss or ectopic expression of Ptch1 confirmed the removal or activation of Hh signaling, respectively, in CNCCs of the mutant embryos (Figs. 3A-F, 4A-D).

At E9.5, Foxc2 is normally expressed in the FNP, BAs, periocular mesenchyme, and cephalic paraxial mesoderm (Fig. 3G,H). Its expression is lost from the medial nasal mesenchyme and the BAs in Wnt1-Cre; Smo ${ }^{n / c} \mathrm{em}-$ bryos (Fig. 3I,J). However, periocular (arrow in Fig. 3I) and lateral nasal mesenchyme (arrow in Fig. 3J) expression is independent of Hh signaling, consistent with these areas being outside of the Shh target field as judged from Ptch1 ${ }^{1 a c Z}$ activity (Fig. 1D,H,Q). As expected, cephalic mesoderm expression (arrowhead in Fig. 3I) is not affected in the mutant because the manipulation was specific to NCCs. Conversely, Wnt1-Cre;R26SmoM2 embryos show clear up-regulation and expansion of Foxc2 expression within ectomesenchyme, providing additional evidence for Foxc2 regulation by the Hh pathway (Fig. 3K,L). Interestingly, even though $\mathrm{Hh}$ signaling is ubiquitously activated in the MNA mesenchyme as indicated by Ptch1 expression (Fig. 3E,F), Foxc2 is excluded from a triangular zone at the midline (Fig. 3L), which suggests that factors other than Hh signaling are also involved in determining the expression domain of Foxc2.

Foxd1 is expressed in the FNP and BAs of the wildtype embryos at E9.5 (Fig. 3M,N), and its expression is lost from both sites when Hh signaling is removed (Fig.
3O,P). Just as with Foxc2, constitutive activation of the Hh pathway in the entire ectomesenchyme leads to the up-regulation and ectopic expression of Foxd1 (Fig. $3 \mathrm{Q}, \mathrm{R}$ ), except in the posterodistal tips of the MNAs (triangle in Fig. 3R). Foxd2, Foxf1, and Foxf2 share an FNP expression domain similar to that of Foxd1, and their expression is also dependent on Hh signaling (Fig. 3S-j). However, these genes show intriguing differences in the MNA. Foxd2 is expressed in the entire MNA, but the expression level increases from distal to proximal (Fig. $3 \mathrm{~S}, \mathrm{~T})$. Its expression is down-regulated upon loss of $\mathrm{Hh}$ responsiveness (Fig. 3U,V). In the Wnt1-Cre;R26SmoM2 MNA, unlike Foxc2 and Foxd1, Foxd2 is not excluded from the posterodistal domain, although the expression is slightly decreased, reflecting its normal gradient (Fig. $3 \mathrm{X})$. The expression of Foxf1 is normally restricted to the distal half of the MNA, and requires Hh signaling (Fig. $3 \mathrm{Y}-\mathrm{b})$. Foxf1 is induced more laterally in the Wnt1Cre;R26SmoM2 MNA (Fig. 3c,d), but not at the very proximal ends (arrow in Fig. 3c). Thus, it exhibits the opposite pattern from Foxc2 and Foxd1. Foxf2 is also expressed in the distal MNA in a Hh-dependent manner, although it does not have a sharp boundary as observed for Foxf1 (Fig. 3e-h). Also unlike Foxf1, Foxf2 is expressed in the entire MNA in Wnt1-Cre;R26SmoM2 embryos (Fig. 3i,j). However, it shows a gradient of expression decreasing from the midline toward the proximal end, which is the opposite of Foxd2.

The expression domains of the Fox genes are further refined by E10.5, highlighting the distinct patterns for each of these genes. In the MNA and the second BA, Foxc2 is expressed strongly around the first pharyngeal cleft (closed arrowheads in Fig. 4E,F), whereas it is repressed in the anterodistal mesenchyme (open arrowhead in Fig. 4F). Foxd1 and Foxd2 are expressed in both of these domains (Fig. 4I,J,M,N). Foxf1 and Foxf2 are expressed at the midline only, as they were at E9.5 (Fig. $4 \mathrm{Q}, \mathrm{R}, \mathrm{U}, \mathrm{V})$. The Foxf1 expression level is high along the entire anteroposterior axis of the MNA (Fig. 4R), whereas Foxf2 expression tapers off in the posterior half (Fig. 4V). The MXA expresses four of the Fox genes examined; Foxd2 and Foxf2 are predominantly expressed rostrally (arrows in Fig. 4N,V), and Foxc2 and Foxd1 in the caudal half (arrows in Fig. 4F,J). All of the five Fox genes are expressed in the medial nasal mesenchyme (Fig. 4F, J,N $, \mathrm{R}, \mathrm{V}$ ), whereas only Foxc2 is detected in the lateral nasal mesenchyme (Fig. 4F). All these expression domains are dependent on $\mathrm{Hh}$ signaling at this stage (Fig. 4E-X), except for that of Foxc2 in the lateral nasal mesenchyme (arrow in Fig. 4H). The normal expression patterns of the Fox genes at E10.5 are summarized in Figure 8B.

\section{Hh signaling specifically regulates Fox gene expression}

Because we observed the changes in Fox gene expression in the mutants as early as E9.5, prior to the onset of a visible phenotype in Wnt1-Cre;Smon/c embryos, it is very likely that these changes are direct and specific responses to the altered Hh signaling. To confirm this, we 

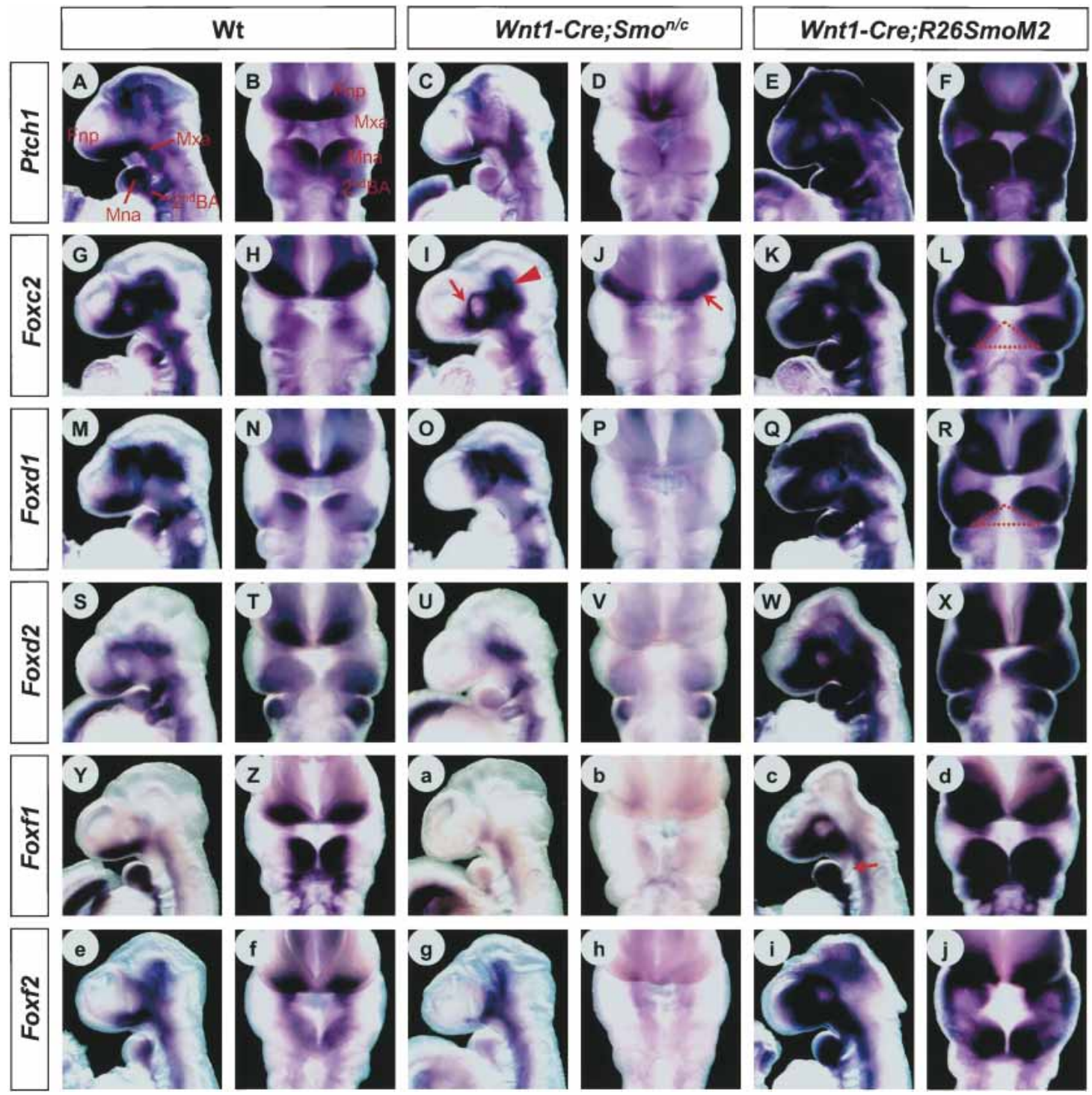

Figure 3. Expression of Fox genes in the facial primordia of E9.5 embryos. Lateral views (odd-number columns) and frontal views (even-number columns) of whole-mount in situ hybridization samples. Genotypes of the embryos and probes as indicated in each panel.

examined the expression of other markers in various domains of the facial mesenchyme.

Spry1, a general target and feedback regulator of receptor tyrosine kinase signaling, is expressed in the mesenchyme around the nasal pits and oral cavity (Fig. 5A; Minowada et al. 1999). Msx1 encodes a homeobox transcription factor that is downstream of BMP signaling in the BAs (Vainio et al. 1993). Msx1 is expressed in most of the FNP, MXA, and the anterodistal MNA at E10.5 (Fig. 5D). dHAND encodes another transcription factor expressed in the distal MNA, under the regulation of endothelin signaling (Fig. 5G; Srivastava et al. 1995; Thomas et al. 1998). Finally, the central mesenchyme of the MNA is labeled by Ptx1, a member of the small bicoid family homeobox genes (Fig. 5J; Lanctôt et al. 1997). All four genes retain their normal expression patterns in
Wnt1-Cre; Smo ${ }^{n / c}$ and Wnt1-Cre;R26SmoM2 embryos (Fig. 5A-L). As mentioned earlier, combinations of Dlx genes determine MNA versus MXA identity within the first BA. The expression of $D l \times 1, D l \times 2, D 1 \times 5$, and $D l \times 6$ is not changed in either of our mutants, indicating that this level of patterning is independent of Hh signaling (Fig. $5 \mathrm{M}-\mathrm{O}$; data not shown).

Because Msx1, dHAND, and Dlx5 expression domains overlap those of the Fox genes shown in Figure 4, their normal expression in Wnt1-Cre; $\mathrm{Smo}^{n / c}$ BAs rules out the possibility that the down-regulation of the Fox genes is secondary to the compromised integrity of the CNCC population in the facial primordia. In addition, the normal expression of Spry1, Msx1, and dHAND, which act as reporters for three other pathways known to be important in craniofacial development, the FGF, BMP and 

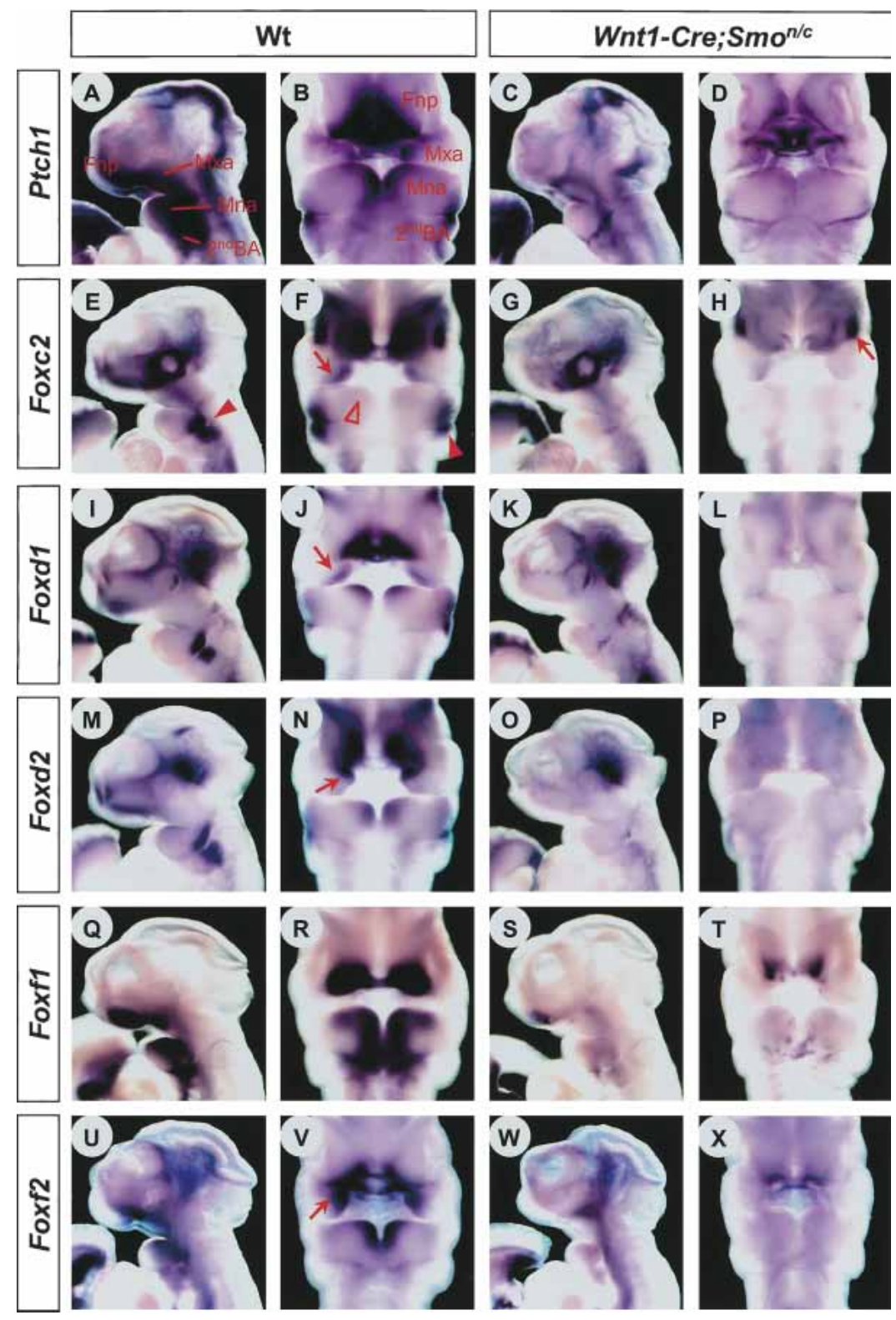

Figure 4. Expression of Fox genes in the facial primordia of E10.5 embryos. Lateral views (oddnumber columns) and frontal views (even-number columns) of whole-mount in situ hybridization samples. Genotypes of the embryos and probes as indicated in each panel.

endothelin pathways, respectively (Kurihara et al. 1994; Barlow and Francis-West 1997; Trumpp et al. 1999|, indicates that these signaling activities are not affected in Wnt1-Cre;Smo ${ }^{n / c}$ embryos. Taken together, these data strongly suggest that altered Hh signaling is directly responsible for the skeletal phenotype and the correlated changes in Fox gene expression we observed.

\section{BA growth defects of Wnt1-Cre; $\mathrm{Smo}^{\mathrm{n} / \mathrm{c}}$ embryos}

Wnt1-Cre;Smo ${ }^{n / c}$ embryos allowed a systematic analysis of the influence of $\mathrm{Hh}$ signaling on the growth of BAs. We compared growth along the mediolateral (M-L, proximodistal) and dorsoventral (D-V) axes of the wildtype and mutant MNAs from E9.5 to E11.5 (Fig. 6A). The wild-type and mutant MNAs have no significant difference in size at E9.5 (Fig. 6A,B,C; M-L: wild type,
$0.917 \pm 0.015 \mathrm{~mm} ;$ mutant, $0.883 \pm 0.015 \mathrm{~mm} ; \quad p=$ 0.130 ; D-V: wild type, $0.403 \pm 0.031 \mathrm{~mm}$, mutant, $0.377 \pm 0.006 \mathrm{~mm} ; p=0.319$ ). However, by E10.5, the mutant MNA is $9 \%$ shorter than the wild type along the M-L axis (Fig. 6B; wild type, $1.903 \pm 0.076 \mathrm{~mm}$; mutant, $1.733 \pm 0.025 \mathrm{~mm} ; p=0.075)$, which is attributable to the deletion of the distal tips, as judged from the expression patterns of $d H A N D, P t \times 1$, and Dlx 5 at this stage (Fig. 5G,H,J,K, M, N; note the loss of dHAND-positive but Ptx1- and Dlx5-negative domain in the Wnt1-Cre;Smo ${ }^{n / c}$ MNA). This deficit does not increase from E10.5 to E11.5 (Fig. 6B). However, although the wild-type MNA undergoes an almost fourfold expansion along the $\mathrm{D}-\mathrm{V}$ axis over this period $(0.247 \pm 0.031 \mathrm{~mm}$ at E10.5, and $0.933 \pm 0.023 \mathrm{~mm}$ at E11.5), that of the mutant MNA is only 1.5 -fold $(0.213 \pm 0.011 \mathrm{~mm}$ at E10.5, and $0.33 \pm 0.035$ $\mathrm{mm}$ at E11.5; Fig. 6C). This difference in growth results 


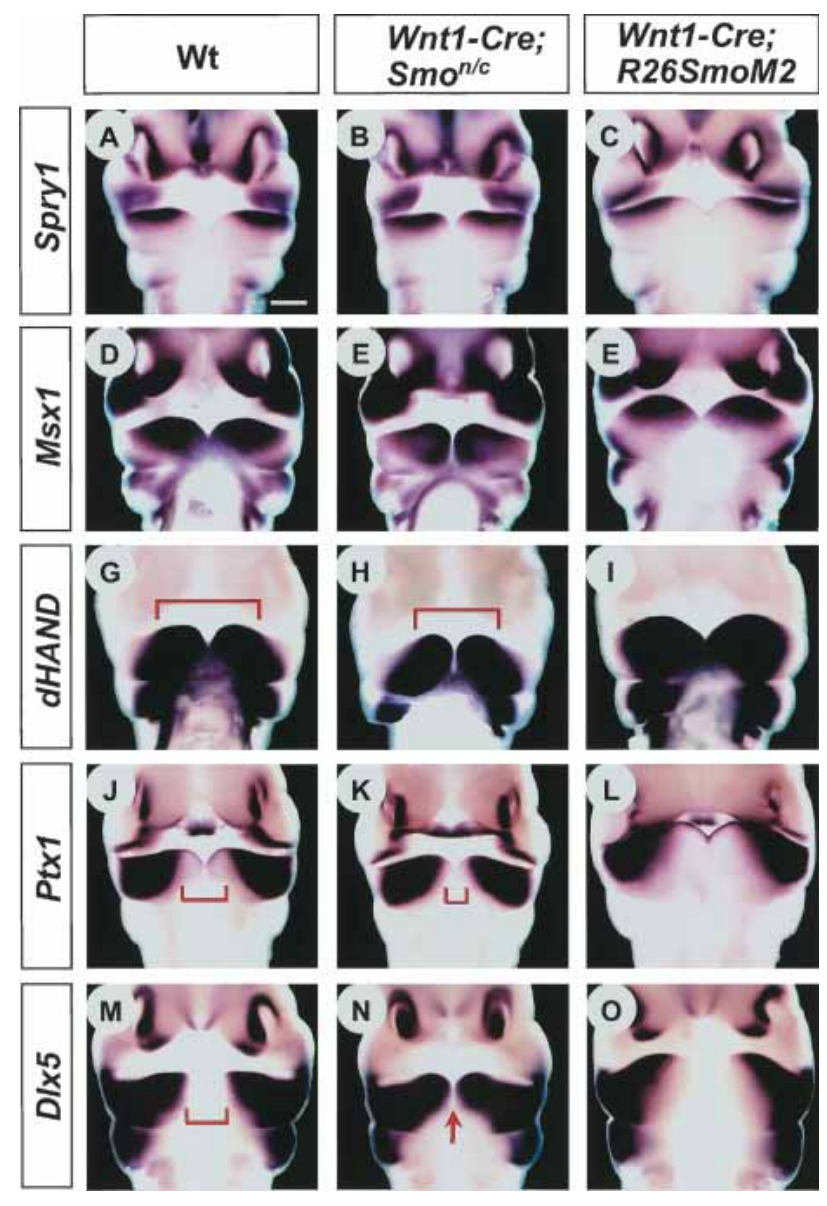

Figure 5. Expression of non-Fox family facial markers in E10.5 embryos. Frontal views of whole-mount in situ hybridization samples. Genotypes of the embryos and probes as indicated in each panel. Bar, $0.25 \mathrm{~mm}$.

in the obvious truncation of the mutant face from E11.5 on (Figs. 6A, 2C-F).

Direct analyses of apoptosis and cell proliferation provide the cellular explanations for the growth phenotype. A cluster of apoptotic cells is found at the midline of the mutant MNAs at E9.5, accounting for the loss of this region over the next day (Fig. 6D-G; density of apoptotic cells for the distal half of the MNA: wild type, $173 \pm 64$ cells $/ \mathrm{mm}^{2}$; mutant, $850 \pm 183$ cells $\left./ \mathrm{mm}^{2}, p=0.010\right)$. At E10.5, apoptosis continues at the midline and is also observed laterally around the first pharyngeal cleft, both in the MNA and second BA mesenchyme (Fig. 6H-K; density of apoptotic cells for the entire MNA: wild type, $71 \pm 21$ cells $/ \mathrm{mm}^{2}$; mutant, $380 \pm 69$ cells $/ \mathrm{mm}^{2}$, $p=0.025)$. The cell proliferation rates are similar between the two genotypes at these stages /density of mitotic cells for the entire MNA: at E9.5, wild type, $372 \pm 64$ cells $/ \mathrm{mm}^{2}$; mutant, $334 \pm 57$ cells $/ \mathrm{mm}^{2} ; p=0.541$; at E10.5, wild type, $299 \pm 19$ cells $/ \mathrm{mm}^{2}$; mutant, $303 \pm 24$ cells $\left./ \mathrm{mm}^{2} ; p=0.844\right)$. By E11.5, apoptosis has mostly stopped in the mutant (data not shown), but instead cell proliferation is decreased throughout the MNA compared with wild-type embryos (Fig. 6L-O). Especially no- ticeable is the difference at the ventromedial tip of the MNA, where the mutant has only $40 \%$ of the wild-type number of dividing cells within the same area (density of mitotic cells for the boxed area: wild type, $408 \pm 102$ cells $/ \mathrm{mm}^{2}$; mutant, $162 \pm 16$ cells $/ \mathrm{mm}^{2} ; \quad p=0.059$ ). These data are in agreement with the observation that the MNA of the wild type, but not the mutant, undergoes rapid outgrowth along the D-V axis around E11.5 (Fig. 6C).

\section{Tongue and lower incisors fail to form in Wnt1-Cre; $\mathrm{Smo}^{\mathrm{n} / \mathrm{c}}$ embryos}

The defects resulting from the removal of Hh signaling are not limited to the skeletal elements. Wnt1-Cre; $\mathrm{Smo}^{n / c}$ embryos also lack a tongue (Fig. 7A,B). The tongue is made of nonneural crest epithelium, mesenchyme containing CNCCs from first, third, and fourth BAs, and muscle cells that have migrated from the occipital somites (Fig. 7A; Kaufman and Bard 1999). The first sign of a defect can be seen at E10.75, even before the tongue starts to grow out in wild-type embryos. At this stage, Myf5- and MyoD-expressing muscle precursors make streams emanating from the somites and accumulating around the midline of the posterior MNAs, where the tongue forms (Fig. 7C; data not shown). These prospective tongue muscle cells are not detected in the mutant MNA (Fig. 7, cf. arrowheads in C and D). Interestingly, the position of the tongue appears to have been specified in the epithelium of the mutant at E11.5, as judged from the expression of Shh, a marker for the tongue epithelium (Fig. 7E,F; Dassule and McMahon 1998). Thus, Hh signaling in the ectomesenchyme may be involved in relaying information from the epithelium to the muscle precursors to coordinate tongue formation.

A paired-box transcription factor $\operatorname{Pax} 9$ prefigures the positions where teeth arise in the first BA mesenchyme at E11.5, and its activity is essential for subsequent development of teeth (Neubüser et al. 1997; Peters et al. 1998). In Wnt1-Cre; $\mathrm{Smo}^{n / c}$ embryos, Pax9 is expressed in the presumptive upper molar, lower molar, and upper incisor, but not the lower incisor, mesenchyme (Fig. $7 \mathrm{G}, \mathrm{H})$. Consistent with this result, the perinatal mutant has one midline upper incisor, one or two upper molars, and two lower molars, but no lower incisor (Fig. 7I-N). The teeth present in the mutant are malformed and arrested, most likely reflecting the role of Hh signaling at later stages of tooth morphogenesis (Dassule et al. 2000). Similar to the tongue, Shh expression in the dental ectoderm is unaltered in the mutant MNA at E11.5 (Fig. $7 \mathrm{E}, \mathrm{F})$, indicating that the primary patterning of the epithelium is independent of Hh signaling within underlying CNCCs.

\section{Discussion}

\section{Extensive requirement for Hh signaling} in craniofacial development

Our study demonstrates that the loss of Hh signaling in NCCs has profound effects on craniofacial structures 
A
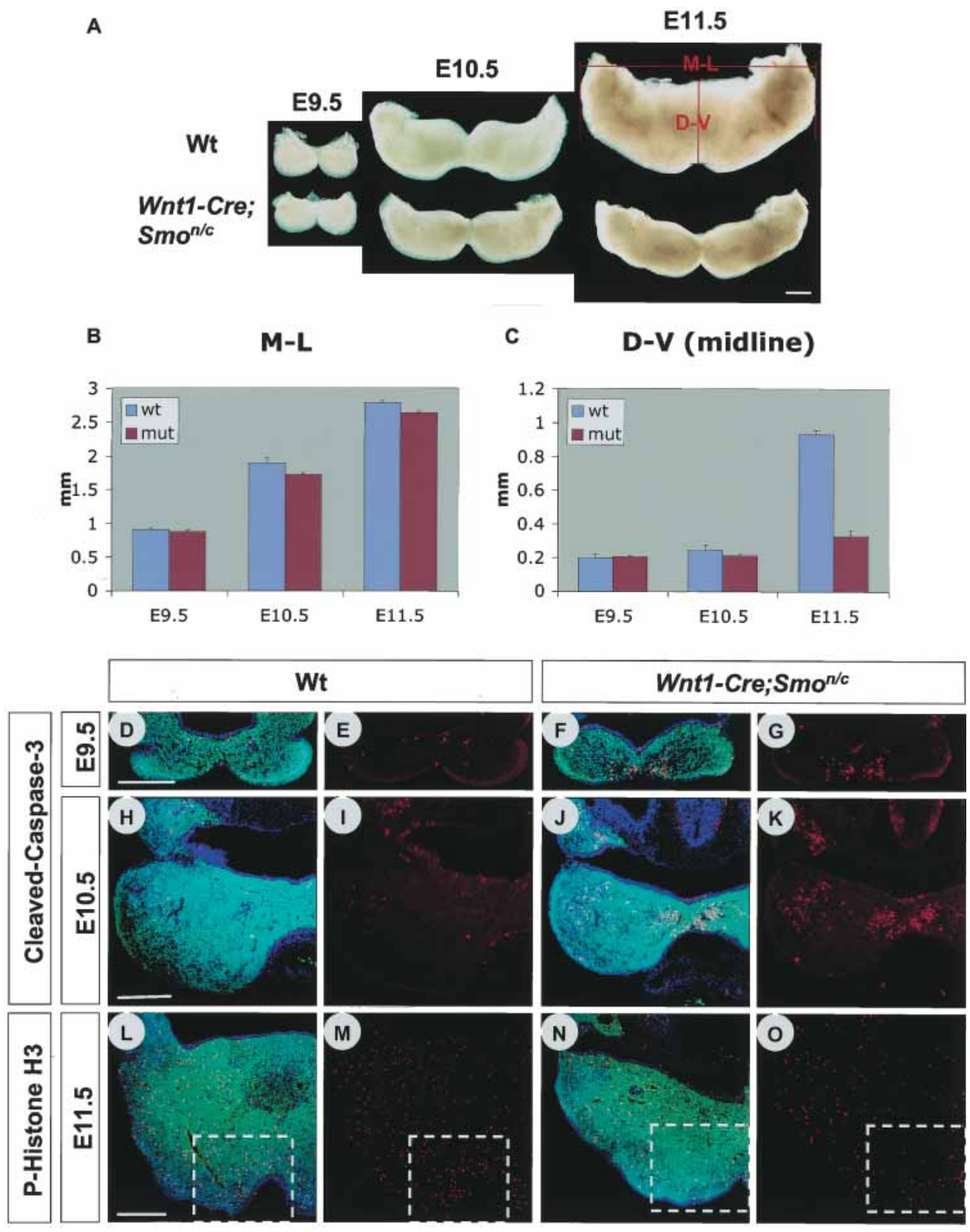

Figure 6. Growth defects in the MNAs of Wnt1-Cre;Smo ${ }^{n / c}$ embryos. (A) MNAs from wild-type (top) and Wnt1-Cre; Smo ${ }^{n / c}(b o t t o m)$ embryos at the stages indicated. $(B, C)$ Quantification of mediolateral $(B)$ and dorsoventral $(C)$ development of the wild-type and Wnt1-Cre;Smo ${ }^{n / c}$ MNAs. Three samples of each genotype were measured at each stage. $(D-O)$ Immunofluorescence with antibodies against cleaved-caspase-3 (red signal; $D-K$ ) and phospho-histone $\mathrm{H} 3$ (red signal; $L-O$ ) to detect apoptotic and mitotic cells, respectively. $(D, E, H, I, L, M)$ Transverse sections through the MNAs of Wnt1-Cre;R26R-YFP embryos. $(F, G, J, K, N, O)$ Transverse sections through the MNAs of Wnt1-Cre;Smo ${ }^{n / c} ; R 26 R-Y F P$ embryos. Stages of development as indicated. Green signal indicates YFP from R26R-YFP; blue signal indicates Topro3 counterstain for nuclei. $(D, H, L, F, J, N)$ Merged images of red, green, and blue channels. $(E, I, M, G, K, O)$ Red channel only. Bar, $0.25 \mathrm{~mm}$.

that are strictly limited to neural crest-derived elements. The defects span both skeletal and nonskeletal components of the head, but notably not the peripheral nervous system, which is also largely derived from NCCs. Based on this and other evidence presented earlier, we conclude that $\mathrm{Hh}$ signaling is dispensable for the generation and migration of NCCs, but is essential for the later steps of craniofacial development involving postmigratory CNCCs at the facial primordia.

Detailed analysis of the Wnt1-Cre;Smon/c head skeleton reveals that all of the NCC-derived elements are absent or reduced, whereas none of the mesodermal ones are affected. The skeletal components in and around the palate (pterygoid, palatine, presphenoid, palatal process 


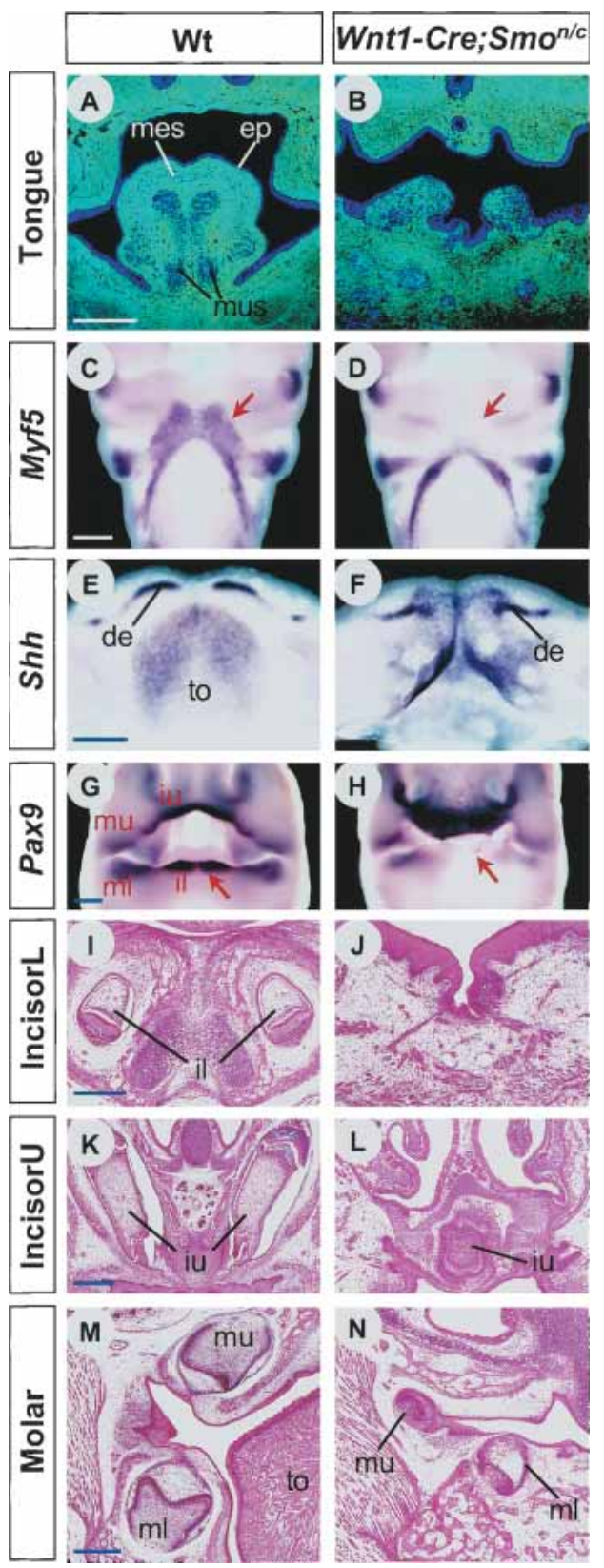

Figure 7. Loss of tongue and lower incisors in Wnt1-Cre; $S m o^{n / c}$ embryos. $(A, B)$ Coronal sections through heads of Wnt1Cre;R26R-YFP $(A)$ and Wnt1-Cre;Smo ${ }^{n / c} ; R 26 R-Y F P(B)$ embryos at E12.5. Green signal indicates YFP from R26R-YFP; blue signal indicates Topro3 counterstain for nuclei. $(\mathrm{C}-\mathrm{H})$ Whole-mount in situ hybridization analyses of tongue and tooth development in wild-type $(C, E, G)$ and Wnt1-Cre;Smo $o^{n / c}(D, F, H)$ embryos. $(C, D)$ Frontal views of Myf5 expression at E10.75. $(E, F)$ Dorsal views of Shh expression in the MNA at E11.5. $(G, H)$ Frontal views of Pax9 expression at E11.5. (I-N) Histological analysis of teeth in coronal sections through heads of wild-type $(I, K, M)$ and Wnt1-Cre;Smo ${ }^{n / c}(J, L, N)$ embryos at E18.5. (de) Dental ectoderm; (ep) tongue epithelium; (il) lower incisor; (iu) upper incisor; (mes) tongue mesenchyme; (ml) lower molar; (mu) upper molar; (mus) tongue muscle; (to) tongue. Bar, $0.25 \mathrm{~mm}$.

of maxilla, vomer) and those in the ear region (gonial bone, tympanic ring, incus, malleus, stapes, styloid pro- cess), as well as the nonskeletal parts such as the tongue and lower incisors, are all completely missing, suggesting that these structures may not be specified in the absence of Hh signaling in NCCs. On the other hand, the dentary, Meckel's cartilage, jugal bone, and zygomatic process of maxilla are present but reduced, which might reflect a direct or indirect influence of Hh signaling on the growth of the BAs. We provide molecular evidence for both cell identity specification and survival/proliferation defects in Wnt1-Cre; Smo ${ }^{n / c}$ facial primordia.

Fox genes as mediators of Hh pathway function during craniofacial development

Our data indicate that $\mathrm{Hh}$ signaling regulates ectomesenchymal expression of five Fox genes, Foxc2, Foxd1, Foxd2, Foxf1, and Foxf2. Although several of these have been reported to be induced by Shh in somites, foregut, or tissue culture (Wu et al. 1998; Furumoto et al. 1999; Mahlapuu et al. 2001a; Ingram et al. 2002), little attention was given to their expression in facial primordia. Consequently, prior to this work, Foxc2 was the only one of these members that had been shown to be transcriptional downstream of Hh signaling in the ectomesenchyme (Yamagishi et al. 2003). Based on our findings, we propose that the Fox genes are the major mediators of the function of Hh signaling in craniofacial morphogenesis. Further support for this model comes from the mutant phenotype of Foxc2 (Fig. 8A; Iida et al. 1997; Winnier et al. 1997). The head skeleton of Foxc2 mutants exhibits defects that overlap those of Wnt1-Cre;Smo ${ }^{n / c}$ mutants, suggesting that the loss of Foxc2 expression can account for at least part of the phenotype of Wnt1Cre; $\mathrm{Smo}^{n / c}$ embryos. In particular, the absence of the palate components (palatal process of the maxilla and palatine) and the middle ear ossicles (incus and stapes) correlates with the expression of Foxc2 in the MXA and second BAs. Foxf2 mutants also have a cleft palate, although this is likely to be secondary to the influence of Foxf2 on tongue morphogenesis (Wang et al. 2003). In contrast, no craniofacial abnormalities were reported in the mutants of either Foxd1 or Foxd2 (Hatini et al. 1996; Kume et al. 2000). This lack of an overt phenotype could be due to a functional redundancy between these or other Fox family members that obscures their importance. Unfortunately, the early lethality caused by mutation of Foxf1 precludes an assessment of its role in facial development (Mahlapuu et al. 2001b).

Although the transcription of Foxc2, Foxd1, Foxd2, Foxf1, and Foxf2 are clearly all under the positive regulation by Hh signaling in facial primordia, the Fox genes are dissimilar from one another in their normal expression patterns (Figs. 3, 4). Furthermore, in the ectomesenchyme of Wnt1-Cre;R26SmoM2 embryos, the distribution and level of each Fox gene transcripts are spatially regulated despite the uniform activation of the Hh pathway (Fig. 3); in the MNA, Foxc2 and Foxd1 are expressed ubiquitously except at the midline, whereas Foxf1 is excluded from the lateral ends. Foxd2 and Foxf2 are both expressed along the entire mediolateral axis, but Foxd2 
A

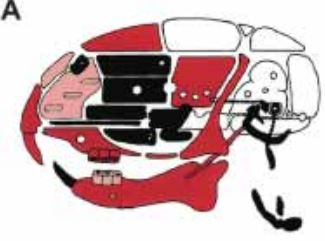

Wnt1-Cre;Smon'c

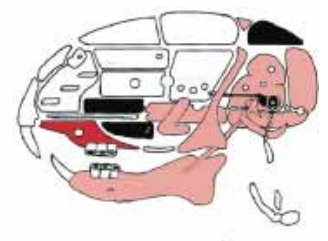

Foxc2 $\%$

B
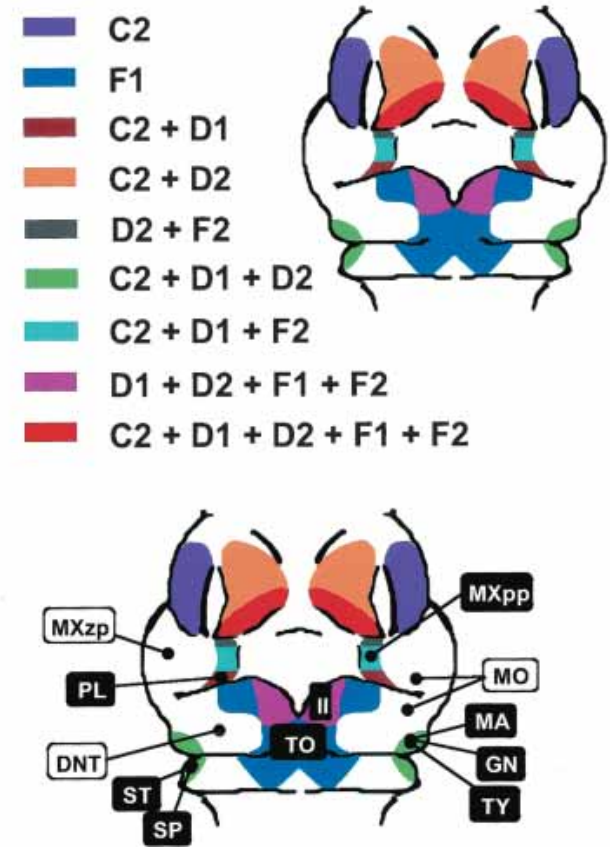

Figure 8. A model of BA patterning by the Shh-Fox genetic pathway. (A) Summary of head skeleton defects of Wnt1-Cre; S$\mathrm{mo}^{n / \mathrm{c}}$ and $\mathrm{Foxc2}^{-/-}$embryos at E18.5. The phenotype of Foxc2 ${ }^{-/-}$ is based on Winnier et al. (1997), which reported more severe defects than Iida et al. (1997). Pink indicates mildly reduced or malformed; red indicates severely reduced; black indicates absent. (B) Summary of the expression patterns of Foxc2, Foxd1, Foxd2, Foxf1, and Foxf2 in wild-type facial primordia at E10.5. $(C)$ The "Fox code map" from $B$, superimposed with the putative fate map of the first and second BAs. The face is symmetric around the midline, although each element is labeled on one side only due to space limitations. (DNT) Dentary; (GN) gonial; (Il) lower incisor; (MA) malleus; (MO) molar; (MXpp) palatal process of maxilla; (MXzp) zygomatic process of maxilla; (PL) palatine; (SP) styloid process; (ST) stapes; (TO) tongue; (TY) tympanic ring. White characters in black box label the elements absent in Wnt1-Cre; Smo ${ }^{n / c}$ embryos, whereas the black characters in white box label those present in the mutant. $A$ was modified with permission from Depew et al. (1999). (c) 1999 The Company of Biologists Limited.

has an increasing, and Foxf2 a decreasing, gradient of intensity from medial to lateral. These observations suggest that Fox genes may be at the regulatory intersection between a Hh pathway input and that of another signaling activity present in a mediolateral gradient in the MNA. For example, if a hypothetical signaling molecule forms an increasing concentration gradient from medial to lateral, then induction of Foxc2, Foxd1, and Foxd2, and repression of Foxf1 and Foxf2 at different thresholds could result in the FOX gene expression patterns described above.

\section{Ectomesenchyme patterning by Fox genes}

How do Fox genes participate in craniofacial development? First, they may be functionally redundant permissive factors that serve common needs of cells such as survival or proliferation. In this case, a certain amount of Fox protein may be required in order for a CNCC to participate in facial morphogenesis, but the exact combination of Fox proteins may not be important. Further, when more than one Fox gene is expressed in the same cell, inactivating one of these may or may not produce abnormalities, depending on its expression level and the sensitivity of the particular cell to the overall Fox gene dosage. Alternatively, certain combinations of Fox genes may have instructive information specifying distinct cell fates. When combined together, the unique expression patterns of each Fox gene make an intriguing map of "Fox codes" in the developing face (Fig. 8B). How these domains defined by different Fox codes correlate with facial structures is not clear, because a fate map of facial development is not yet available. However, if one assumes that the relative positions of the facial element precursor domains at E10.5 are the same as those of the facial elements in the newborns (Fig. 8C), this leads to some interesting predictions. For example, the mesenchyme around the first pharyngeal cleft is expected to make the skeleton associated with the otic capsule, such as stapes, malleus, gonial bone, and tympanic ring. This mesenchyme expresses Foxc2 + Foxd1 + Foxd2. On the other hand, the tongue arises at the midline of the MNAs, where Foxf1, but none of these three Fox genes, is expressed. The domain anterior to the tongue, where the lower incisors form, has still another Fox code, Foxd1 + Foxd2 + Foxf1 + Foxf2. All these facial structures are lost in Wnt1-Cre;Smo ${ }^{n / c}$ embryos, consistent with all the Fox codes being lost. The absence of craniofacial defects in Foxd1 or Foxd2 mutants (Hatini et al. 1996; Kume et al. 2000) could be explained by some degree of tolerance in the Fox codes, which would allow more than one combination to encode the same element. The molars and the body of the dentary apparently develop outside of the Fox gene expression domains. Accordingly, they are present in Wnt1-Cre;Smo ${ }^{n / c}$ heads, suggesting that they are specified by other mechanisms. Similar correlations can be found for the MXA-derived elements, but not for the FNP-derived ones. The FNP derivatives (nasal bone, nasal cartilage, premaxilla, and upper incisor) suffer relatively mild defects in Wnt1Cre; $\mathrm{Smo}^{n / c}$ embryos, where none of them are completely lost. Furthermore, no defects in these structures are observed in Foxc2 ${ }^{-/-}$embryos (Iida et al. 1997; Winnier et al. 1997). Therefore, we speculate that unlike the first and second BAs, specification of individual skeletal elements in the FNP is independent of Hh signaling or Fox gene expression in the ectomesenchyme, though FNP growth is dependent on Hh signaling (Fig. 2A-F). Clearly, distinguishing between the two models for Fox gene 
function will require additional loss-of-function, gain-offunction, and gene swapping experiments.

\section{Hh signaling and growth of facial primordia}

Wnt1-Cre;Smon/c MNAs suffer high levels of apoptosis at E9.5 and E10.5, and then decreased proliferation at E11.5, resulting in severe growth deficiency (Fig. 6). This observation that $\mathrm{Hh}$ signaling regulates growth of facial primordia in mouse embryos is in line with previous reports on the role of Shh during craniofacial development of chick (Ahlgren and Bronner-Fraser 1999; Hu and Helms 1999); inhibition of Shh function during migration of NCCs into BAs (stage 10, equivalent to E8.5 of mouse) leads to a reduction in the size of head in $24 \mathrm{~h}$ (i.e., equivalent to E9.5 of mouse; Ahlgren and BronnerFraser 1999). Although the cell proliferation rate did not change, apoptosis in NCCs increased, indicating that enhanced cell death underlay this defect. In another study, Shh protein was applied ectopically in the FNP at a later stage (stage 20, equivalent to E10.5 of mouse), resulting in a mediolateral expansion of the FNP (Hu and Helms 1999). This phenotype was attributed to increase in cell proliferation, which was obvious by $20 \mathrm{~h}$ after the addition of the protein (i.e., equivalent to E11.5 of mouse). Our mouse studies reported here agree with these findings that Shh supports cell survival during early stages and promotes proliferation at later stages to control the size of facial primordia. However, there is one significant difference between the mouse and chick results; although blocking Shh function causes a clear reduction in head size in the chick at a stage equivalent to E9.5 of mouse development (Ahlgren and Bronner-Fraser 1999), Wnt1-Cre; $\mathrm{Smo}^{n / c}$ facial primordia have no noticeable growth defects until E10.5 (Figs. 2A,B, 6A-C). This difference appears to stem from the fact that the manipulation of chick embryos affected the survival of migrating CNCCs (Ahlgren and Bronner-Fraser 1999), whereas in Wnt1-Cre;Smo ${ }^{n / c}$ embryos the deleterious effects are limited to postmigratory CNCCs, based on our analysis (Fig. 2). Although several explanations are possible for these differences, the most plausible one focuses on the experimental difference, where inhibition of Shh protein in the chick experiments eliminates Hh signaling within all cell types in that area, that is, epithelial cells, mesodermal cells, and NCCs, not simply NCCs as in Wnt1Cre; $\mathrm{Smo}^{n / c}$ embryos. In this case, the observed apoptosis in migrating CNCCs in the chick may be an indirect consequence of disrupting Shh signaling in another cell population. The failure to detect Ptch1 up-regulation in migrating CNCCs in either the chick or mouse (Ahlgren and Bronner-Fraser 1999; J. Jeong, T. Tenzen, and A. McMahon, unpubl.) suggests that these cells are not in fact directly responding to Hh signaling.

\section{Materials and methods}

Generation of R26SmoM2 line and neural crest-specific mutants of Hh signaling

SmoM2 contains a point mutation, W539L, which renders it constitutively active (Xie et al. 1998). A cDNA encoding
SmoM2 with yellow fluorescent protein (EYFP, Clontech) fused at its $\mathrm{C}$ terminus was cloned into the BigT vector (Srinivas et al. 2001). The resulting plasmid was digested with PacI and AscI to release the floxed neo/4xpA-SmoM2-YFP cassette, which was inserted into pROSA26PA (Srinivas et al. 2001). This targeting vector was linearized by SwaI digestion and electroporated into AV3 embryonic stem cells. After G418 selection, recombinants at the ROSA26 (R26) locus were identified by PCR and Southern blot hybridization (Soriano 1999), and the chimeras were generated by blastocyst injection. Subsequent PCR genotyping of the mice carrying this allele was performed on tail biopsies (Soriano 1999).

Wnt1-Cre, Smo null $\left(S m o^{n}\right)$, and Smo conditional $\left(S m o^{c}\right)$ alleles have been described (Danielian et al. 1998; Long et al. 2001; Zhang et al. 2001). Wnt1-Cre; Smo ${ }^{n / c}$ embryos were obtained from the crosses of Wnt1-Cre;Smo ${ }^{n /+} ; R 26 R-L a c Z$ or Wnt1-Cre;Smo ${ }^{n /+} ;$ R26R-YFP males (R26R-LacZ and R26R-YFP are Cre reporters; Soriano 1999; Srinivas et al. 2001) and $S m o^{c / c}$ females. Wnt1-Cre;R26SmoM2 embryos were generated by mating Wnt1-Cre animals with R26SmoM2 mice. For both crosses, embryos with genotypes other than Wnt1-Cre; Smo ${ }^{n / c}$ or Wnt1-Cre;R26SmoM2 were phenotypically wild type, and so used as controls for the experiments and referred to as "wild type" in the text.

$\beta$-galactosidase analysis for Shh ${ }^{\mathrm{LacZ}}$, Ptch $1^{\mathrm{LacZ}}$, and R26R-LacZ Cre reporter lines

The $S h h^{\text {LacZ }}$ allele will be described elsewhere (A. Kottmann and T. Jessell, in prep.). Ptch1 $1^{\text {LacZ/+ }}$ and R26R-LacZ lines have been described (Goodrich et al. 1997; Soriano 1999). Wholemount detection of $\beta$-galactosidase activity was done using Xgal as described (Whiting et al. 1991). Following the staining, $\mathrm{Shh}^{\mathrm{LacZ/+}}$ and Ptch1 $1^{\mathrm{LacZ} /+}$ embryos were embedded in $15 \%$ gelatin and sectioned by vibratome at $50 \mu \mathrm{m}$ (E9.5 and E10.5) or 100 $\mu \mathrm{m}$ (E12.5). To visualize the distribution of NCCs on sections using Wnt1-Cre;R26R-LacZ genotype, the embryos were fixed in $4 \%$ paraformaldehyde in phosphate-buffered saline (PBS) for 2-5 h, washed in PBS three times for 15 min each, cryoprotected in $30 \%$ sucrose, $0.1 \mathrm{M}$ phosphate buffer $(\mathrm{pH} 7.4$ ) overnight, and embedded in OCT compound (Tissue-Tek). Frozen sections were prepared at $12 \mu \mathrm{m}$ (E9.5 and E10.5) or $24 \mu \mathrm{m}$ (E12.5) and subject to Xgal staining. Nuclear fast red (Sigma) was used as counterstain.

In situ hybridization, immunohistochemistry, skeletal preparation, and histology

Whole-mount digoxigenin in situ hybridization was performed as described (Wilkinson and Nieto 1993). Immunostaining for the neurofilament was performed as described (Mark et al. 1993) using a monoclonal antibody $(2 \mathrm{H} 3$, Developmental Studies Hybridoma Bank) at a 1:20 dilution. Skeleton was prepared as described (Wallin et al. 1994), except that the embryos were stained at $37^{\circ} \mathrm{C}$ for $4 \mathrm{~d}$. For the histological examination of teeth, E18.5 embryos were fixed in Bouin's fixative, dehydrated in ethanol and xylene, and embedded in paraffin. Sections were cut at $6 \mu \mathrm{m}$ and stained with hematoxylin and eosin.

Analysis of apoptosis and cell proliferation

Apoptosis and cell proliferation were detected using rabbit anticleaved-caspase-3 antibody (Cell Signaling Technology) and anti-phospho-histone $\mathrm{H} 3$ antibody (Upstate Biochem), respectively. For immunofluorescence, frozen sections were prepared at $12 \mu \mathrm{m}$ as described above for the section Xgal staining. After 
blocking the sections in $3 \%$ bovine serum albumin, $1 \%$ heatinactivated sheep serum, $0.1 \%$ Triton X-100 in PBS for $1 \mathrm{~h}$, primary antibodies were applied at $4{ }^{\circ} \mathrm{C}$ overnight, followed by secondary antibody (Alexa568 goat anti-rabbit, Molecular Probes) at room temperature for $1 \mathrm{~h}$. The sections were also stained for nuclei with Topro3 (Molecular Probes). The images were collected using a Zeiss LSM510 confocal microscope, and apoptotic or mitotic cells were counted manually from these images. Data from three embryos for each genotype were used for statistical analysis.

\section{Acknowledgments}

We are grateful to Tom Jessell for permission to use the $S h h^{1 a c z}$ strain, which was generated in his laboratory, prior to publication. We also thank Thomas Lufkin for providing plasmids for in situ probes, Jing Yu and Tom Carroll for sharing reagents, and Steve Vokes for critical reading of the manuscript. J.M. is a fellow of the Damon Runyon Cancer Research Foundation (DRG-1750-03). This work was funded by grants from NIH NS33642 and DK56246.

The publication costs of this article were defrayed in part by payment of page charges. This article must therefore be hereby marked "advertisement" in accordance with 18 USC section 1734 solely to indicate this fact.

\section{References}

Ahlgren, S.C. and Bronner-Fraser, M. 1999. Inhibition of Sonic hedgehog signaling in vivo results in craniofacial neural crest cell death. Curr. Biol. 9: 1304-1314.

Barlow, A.J. and Francis-West, P.H. 1997. Ectopic application of recombinant $\mathrm{BMP}-2$ and $\mathrm{BMP}-4$ can change patterning of developing chick facial primordia. Development 124: 391398.

Beverdam, A., Merlo, G.R., Paleari, L., Mantero, S., Genova, F., Barbieri, O., Janvier, P., and Levi, G. 2002. Jaw transformation with gain of symmetry after $D 1 \times 5 / D 1 \times 6$ inactivation: Mirror of the past? Genesis 34: 221-227.

Carlsson, P. and Mahlapuu, M. 2002. Forkhead transcription factors: Key players in development and metabolism. Dev. Biol. 250: 1-23.

Chai, Y., Jiang, X., Ito, Y., Bringas, P., Han, J., Rowitch, D.H., Soriano, P., McMahon, A.P., and Sucov, H.M. 2000. Fate of the mammalian cranial neural crest during tooth and mandibular morphogenesis. Development 127: 1671-1679.

Chiang, C., Litingtung, Y., Lee, E., Young, K.E., Corden, J.L., Westphal, H., and Beachy, P. 1996. Cyclopia and defective axial patterning in mice lacking Sonic hedgehog gene function. Nature 383: 407-413.

Clevidence, D.E., Overdier, D.G., Peterson, R.S., Porcella, A., Ye, H., Paulson, K.E., and Costa, R.H. 1994. Members of the HNF3/forkhead family of transcription factors exhibit distinct cellular expression patterns in lung and regulate the surfactant protein B promoter. Dev. Biol. 166: 195-209.

Couly, G., Creuzet, S., Bennaceur, S., Vincent, C., and Le Douarin, N.M. 2002. Interaction between Hox-negative cephalic neural crest cells and the foregut endoderm in patterning the facial skeleton in the vertebrate head. Development 129: 1061-1073.

Danielian, P.S., Muccino, D., Rowitch, D.H., Michael, S.K., and McMahon, A.P. 1998. Modification of gene activity in mouse embryos in utero by a tamoxifen-inducible form of Cre recombinase. Curr. Biol. 8: 1323-1326.
Dassule, H.R. and McMahon, A.P. 1998. Analysis of epithelialmesenchymal interactions in the initial morphogenesis of the mammalian tooth. Dev. Biol. 202: 215-227.

Dassule, H.R., Lewis, P., Bei, M., Maas, R., and McMahon, A.P. 2000. Sonic hedgehog regulates growth and morphogenesis of the tooth. Development 127: 4775-4785.

Depew, M.J., Liu, J.K., Long, J.E., Presley, R., Meneses, J.J., Pedersen, R.A., and Rubenstein, J.L.R. 1999. Dlx5 regulates regional development of the branchial arches and sensory capsules. Development 126: 3831-3846.

Depew, M.J., Tucker, A.S., and Sharpe, P.T. 2002a. Craniofacial development. In Mouse development, patterning, morphogenesis, and organogenesis (eds. J. Rossant and P.P.L. Tam), pp. 421-498. Academic Press, San Diego, CA.

Depew, M.J., Lufkin, T., and Rubenstein, J.L.R. 2002b. Specification of jaw subdivisions by Dlx genes. Science 298: 381385.

Echelard, Y., Epstein, D.J., St-Jacques, B., Shen, L., Mohler, J., McMahon, J.A., and McMahon, A.P. 1993. Sonic Hedgehog, a member of a family of putative signaling molecules, is implicated in the regulation of CNS polarity. Cell 75: $1417-$ 1430.

Echelard, Y., Vassileva, G., and McMahon, A.P. 1994. Cis-acting regulatory sequences governing Wnt-1 expression in the developing mouse CNS. Development 120: 2213-2224.

Ferguson, C.A., Tucker, A.S., and Sharpe, P.T. 2000. Temporospatial cell interactions regulating mandibular and maxillary arch patterning. Development 127: 403-412.

Furumoto, T., Miura, N., Akasaka, T., Mizutani-Koseki, Y., Sudo, H., Fukuda, K., Maekawa, M., Yuasa, S., Fu, Y., Moriya, H., et al. 1999. Notochord-dependent expression of MFH1 and PAX1 cooperates to maintain the proliferation of sclerotome cells during the vertebral column development. Dev. Biol. 210: 15-29.

Gammill, L.S. and Bronner-Fraser, M. 2003. Neural crest specification: Migration into genomics. Nat. Rev. Neurosci. 4: 795-805.

Gendron-Maguire, M., Mallo, M., Zhang, M., and Gridley, T. 1993. Hoxa2 mutant mice exhibit homeotic transformation of skeletal elements derived from cranial neural crest. Cell 75: 1317-1331.

Goodrich, L.V., Johnson, R.L., Milenkovic, L., McMahon, J.A., and Scott, M.P. 1996. Conservation of the hedgehog/patched signaling pathway from flies to mice: Induction of a mouse patched gene by Hedgehog. Genes \& Dev. 10: 301-312.

Goodrich, L.V., Milenkovic, L., Higgins, K.M., and Scott, M.P. 1997. Altered neural cell fates and medulloblastoma in mouse patched mutants. Science 277: 1109-1113.

Grammatopoulos, G.A., Bell, E., Toole, L., Lumsden, A., and Tucker, A.S. 2000. Homeotic transformation of branchial arch identity after Hoxa2 overexpression. Development 127: 5355-5365.

Hatini, V., Tao, W., and Lai, E. 1994. Expression of winged helix genes, BF-1 and BF-2 define adjacent domains within the developing forebrain and retina. J. Neurobiol. 25: 1293-1309.

Hatini, V., Huh, S.O., Herzlinger, D., Soares, V.C., and Lai, E. 1996. Essential role of stromal mesenchyme in kidney morphogenesis revealed by targeted disruption of winged helix transcription factor BF-2. Genes \& Dev. 10: 1467-1478.

Helms, J.A. and Schneider, R.A. 2003. Cranial skeletal biology. Nature 423: 326-331.

Hu, D. and Helms, J.A. 1999. The role of Sonic hedgehog in normal and abnormal craniofacial morphogenesis. Development 126: 4873-4884.

Hu, D., Marcucio, R.S., and Helms, J.A. 2003. A zone of frontonasal ectoderm regulates patterning and growth in the 
face. Development 130: 1749-1758.

Iida, K., Koseki, H., Kakinuma, H., Kato, N., Mizutani-Koseki, Y., Ohuchi, H., Yoshioka, H., Noji, S., Kawamura, K., Kataoka, Y., et al. 1997. Essential roles of the winged helix transcription factor MFH-1 in aortic arch patterning and skeletogenesis. Development 124: 4627-4638.

Ingram, W.J., Wicking, C.A., Grimmond, S.M., Forrest, A.R., and Wainwright, B.J. 2002. Novel genes regulated by Sonic Hedgehog in pluripotent mesenchymal cells. Oncogene 21: 8196-8205.

Jiang, X., Rowitch, D.H., Soriano, P., McMahon, A.P., and Sucov, H.M. 2000. Fate of the mammalian cardiac neural crest. Development 127: 1607-1616.

Kaufman, M.H. and Bard, J.B.L. 1999. The anatomical basis of mouse development, pp. 162-165. Academic Press, San Diego, CA.

Köntges, G. and Lumsden, A. 1996. Rhombencephalic neural crest segmentation is preserved throughout craniofacial ontogeny. Development 122: 3229-3242.

Kronmiller, J.E. and Nguyen, T. 1996. Spatial and temporal distribution of Indian Hedgehog mRNA in the embryonic mouse mandible. Arch. Oral Biol. 41: 577-583.

Kume, T., Deng, K., and Hogan, B.L.M. 2000. Minimal phenotype of mice homozygous for a null mutation in the forkhead/winged helix gene, MF2. Mol. Cell. Biol. 20: 14191425.

Kurihara, Y., Kurihara, H., Suzuki, H., Kodama, T., Maemura, K., Nagai, R., Oda, H., Kuwaki, T., Cao, W.H., Kamada, N., et al. 1994. Elevated blood pressure and craniofacial abnormalities in mice deficient in endothelin-1. Nature 368: 703710.

Lanctôt, C., Lamolet, B., and Drouin, J. 1997. The bicoid-related homeoprotein Ptx1 defines the anterior domain of the embryo and differentiates posterior from anterior lateral mesoderm. Development 124: 2807-2817.

Lee, S.-H., Fu, K.K., Hui, J.N., and Richman, J.M. 2001. Noggin and retinoic acid transform the identity of avian facial prominences. Nature 414: 909-912.

Long, F., Zhang, X.M., Karp, S., Yang, Y., and McMahon, A.P. 2001. Genetic manipulation of hedgehog signaling in the endochondral skeleton reveals a direct role in the regulation of chondrocyte proliferation. Development 128: 5099-5108.

Mahalpuu, M., Enerbäck, S., and Carlsson, P. 2001a. Haploinsufficiency of the forkhead gene Foxf1, a target for sonic hedgehog signaling, causes lung and foregut malformations. Development 128: 2397-2406.

Mahlapuu, M., Ormestad, M., Enerbäck, S., and Carlsson, P. 2001b. The forkhead transcription factor FoxF1 is required for differentiation of extraembryonic and lateral plate mesoderm. Development 128: 155-166.

Mark, M., Lufkin, T., Vonesch, J.-L., Ruberte, E., Olivo, J.-C., Dolle, P., Gorry, P., Lumsden, A., and Chambon, P. 1993. Two rhombomeres are altered in Hoxa-1 mutant mice. Development 119: 319-338.

McMahon, A.P., Ingham, P.W., and Tabin, C.J. 2003. Developmental roles and clinical significance of Hedgehog signaling. Curr. Top. Dev. Biol. 53: 1-114.

Minowada, G., Jarvis, L.A., Chi, C.L., Neubüser, A., Sun, X., Hacohen, N., Krasnow, M.A., and Martin, G. 1999. Vertebrate Sprouty genes are induced by FGF signaling and can cause chondrodysplasia when overexpressed. Development 126: 4465-4475.

Mitchell, P.J., Timmons, P.M., Hebert, J.M., Rigby, P.W., and Tjian, R. 1991. Transcription factor AP-2 is expressed in neural crest cell lineages during mouse embryogenesis. Genes \& Dev. 5: 105-119.
Miura, N., Wanaka, A., Tohyama, M., and Tanaka, K. 1993. MFH-1, a new member of the fork head domain family, is expressed in developing mesenchyme. FEBS. 326: 171-176.

Miura, N., Kakinuma, H., Sato, M., Aiba, N., Terada, K., and Sugiyama, T. 1998. Mouse forkhead (winged helix) gene LUN encodes a transactivator that acts in the lung. Genomics 50: 346-356.

Neubüser, A., Peters, H., Balling, R., and Martin, G.R. 1997. Antagonistic interactions between FGF and BMP signaling pathways: A mechanism for positioning the sites of tooth formation. Cell 90: 247-255.

Noden, D.M. 1983. The role of the neural crest in patterning of avian cranial skeletal, connective, and muscle tissues. Dev. Biol. 96: 144-165.

Osumi-Yamashita, N., Ninomiya, Y., Doi, H., and Eto, K. 1994. The contribution of both forebrain and midbrain crest cells to the mesenchyme in the frontonasal mass of mouse embryo. Dev. Biol. 164: 409-419.

Pasqualetti, M., Ori, M., Nardi, I., and Rijli, F.M. 2000. Ectopic Hoxa2 induction after neural crest migration results in homeosis of jaw elements in Xenopus. Development 127: 5367-5378.

Pera, E.M. and Kessel, M. 1997. Patterning of the chick forebrain anlage by the prechordal plate. Development 124: 41534162.

Peters, H., Neubüser, A., Kratochwil, K., and Balling, R. 1998. Pax9-deficient mice lack pharyngeal pouch derivatives and teeth and exhibit craniofacial and limb abnormalities. Genes \& Dev. 12: 2735-2747.

Rijli, F.M., Mark, S.L., Dierich, A., Dollé, P., and Chambon, P. 1993. A homeotic transformation is generated in the rostral branchial region of the head by disruption of Hoxa2, which acts as a selector gene. Cell 75: 1333-1349.

Roessler, E., Belloni, E., Gaudenz, K., Jay, P., Berta, P., Scherer, S.W., Tsui, L.-C., and Muenke, M. 1996. Mutations in the human Sonic hedgehog gene cause holoprosencephaly. Nat. Genet. 14: 357-360.

Santagati, F. and Rijli, F.M. 2003. Cranial neural crest and the building of the vertebrate head. Nat. Rev. Neurosci. 4: 806818.

Sasaki, H. and Hogan, B.L.M. 1993. Differential expression of multiple fork head related genes during gastrulation and axial pattern formation in the mouse embryo. Development 118: 47-59.

Soriano, P. 1999. Generalized lacZ expression with the ROSA26 Cre reporter strain. Nat. Genet. 21: 70-71.

Srinivas, S., Watanabe, T., Lin, C.-S., William, C.M., Tanabe, Y., Jessell, T.M., and Costantini, F. 2001. Cre reporter strains produced by targeted insertion of EYFP and ECFP into the ROSA26 locus. BMC Dev. Biol. 1: 4.

Srivastava, D., Cserjesi, P., and Olson, E.N. 1995. A subclass of bHLH proteins required for cardiac morphogenesis. Science 270: 1995-1999.

St-Jacques, B., Hammerschmidt, M., and McMahon, A.P. 1999. Indian hedgehog signaling regulates proliferation and differentiation of chondrocytes and is essential for bone formation. Genes \& Dev. 13: 2072-2086.

Thomas, T., Kurihara, H., Yamagishi, H., Kurihara, Y., Yazaki, Y., Olson, E.N., and Srivastava, D. 1998. A signaling cascade involving endothelin-1, dHAND and Msx1 regulates development of neural-crest-derived branchial arch mesenchyme. Development 125: 3005-3014.

Trainor, P. and Krumlauf, R. 2000. Plasticity in mouse neural crest cells reveals a new patterning role for cranial mesoderm. Nat. Cell Biol. 2: 96-102.

Trainor, P.A., Ariza-McNaughton, L., and Krumlauf, R. 2002. 
Role of the isthmus and FGFs in resolving the paradox of neural crest plasticity and prepatterning. Science 295: 12881291.

Trumpp, A., Depew, M.J., Rubenstein, J.L.R., Bishop, J.M., and Martin, G.R. 1999. Cre-mediated gene inactivation demonstrates that FGF8 is required for cell survival and patterning of the first branchial arch. Genes \& Dev. 13: 3136-3148.

Vainio, S., Karavanova, I., Jowett, A., and Thesleff, I. 1993. Identification of BMP-4 as a signal mediating secondary induction between epithelial and mesenchymal tissues during early tooth development. Cell 75: 45-58.

Wallin, J., Wilting, J., Koseki, H., Fritsch, R., Christ, B., and Balling, R. 1994. The role of Pax-1 in axial skeleton development. Development 120: 1109-1121.

Wang, T., Tamakoshi, T., Uezato, T., Shu, F., Kanzaki-Kato, N., Fu, Y., Koseki, H., Yoshida, N., Sugiyama, T., and Miura, N. 2003. Forkhead transcription factor Foxf2 (LUN)-deficient mice exhibit abnormal development of secondary palate. Dev. Biol. 259: 83-94.

Whiting, J., Marshall, H., Cook, M., Krumlauf, R., Rigby, P.W., Scott, D., and Allemann, R.K.. 1991. Multiple spatially specific enhancers are required to reconstruct the pattern of Hox-2.6 gene expression. Genes \& Dev. 5: 2048-2059.

Wilkinson, D.G. and Nieto, M.A. 1993. Detection of messenger RNA by in situ hybridization to tissue sections and whole mounts. Methods Enzymol. 225: 361-373.

Winnier, G.E., Hargett, L., and Hogan, B.L.M. 1997. The winged helix transcription factor $M F H 1$ is required for proliferation and patterning of paraxial mesoderm in the mouse embryo. Genes \& Dev. 11: 926-940.

Wu, S.C.-Y., Grindley, J., Winnier, G., Hargett, L., and Hogan, B.L.M. 1998. Mouse Mesenchyme forkhead 2 (Mf2): Expression, DNA binding and induction by sonic hedgehog during somitogenesis. Mech. Dev. 70: 3-13.

Xie, J., Murone, M., Luoh, S., Ryan, A., Gu, Q., Zhang, C., Bonifas, J.M., Lam, C.-W., Hynes, M., Goddard, A., et al. 1998. Activating Smoothened mutations in sporadic basal-cell carcinoma. Nature 391: 90-92.

Yamagishi, H., Maeda, J., Hu, T., McAnally, J., Conway, S.J., Kume, T., Meyers, E.N., Yamagishi, C., and Srivastava, D. 2003. $T b \times 1$ is regulated by tissue-specific forkhead proteins through a common Sonic hedgehog-responsive enhancer. Genes \& Dev. 17: 269-281.

Zhang, X.M., Ramalho-Santos, M., and McMahon, A.P. 2001. Smoothened mutants reveal redundant roles for Shh and thh signaling including regulation of $\mathrm{L} / \mathrm{R}$ symmetry by the mouse node. Cell 106: 781-792. 


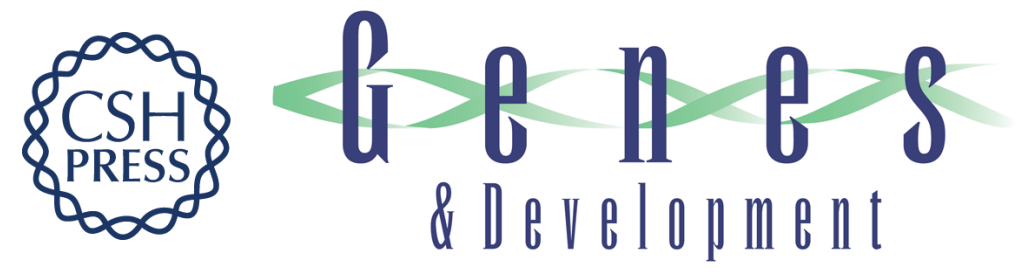

\section{Hedgehog signaling in the neural crest cells regulates the patterning and growth of facial primordia}

Juhee Jeong, Junhao Mao, Toyoaki Tenzen, et al.

Genes Dev. 2004, 18:

Access the most recent version at doi:10.1101/gad.1190304

\section{Supplemental http://genesdev.cshlp.org/content/suppl/2004/04/05/18.8.937.DC1 Material}

References This article cites 70 articles, 35 of which can be accessed free at: http://genesdev.cshlp.org/content/18/8/937.full.html\#ref-list-1

\section{License}

Email Alerting

Receive free email alerts when new articles cite this article - sign up in the box at the top Service

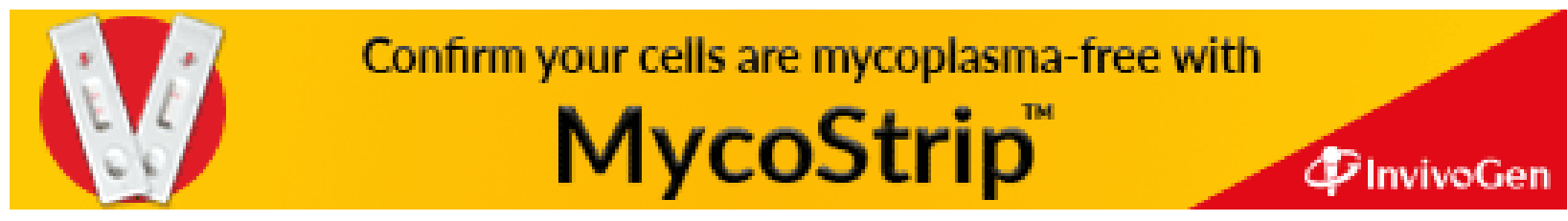

